\title{
A 50 AÑOS DEL INICIO DE LA REVISTA CUBANA PENSAMIENTO CRÍTICO: una ventana a la herejía y la tradición revolucionaria cubana ${ }^{{ }^{*}}$
}

\author{
50 YEARS FROM THE START OF THE CUBAN \\ MAGAZINE PENSAMIENTO CRÍTICO: \\ a window on Cuban heresy and revolucionary tradition
}

\author{
Magdiel Sánchez Quiroz
}

\section{Resumen}

El artículo se concentra en el estudio de los aportes teóricos que tuvo la revista cubana Pensamiento Crítico a 50 años de su primer número. Analiza sus aportes a la teoría crítica en Latinoamérica y sus relaciones con el proceso del que surge: la Revolución cubana. Esto último no es sencillo, si se toma en cuenta que la revista fue cerrada por órdenes de la máxima autoridad del país luego de 5 años de trabajo. Analiza a partir de ocho rasgos a la revista en tanto praxis política y teórica que le dan su carácter original e inédito y el cómo desde ellos, se asume e inscribe en la tradición y herejía revolucionarias de la que formaron parte Fidel Castro Ruz y Ernesto "Che” Guevara.

Palabras clave: Revolución Cubana, Teoría Crítica, Izquierda Latinoamericana

\begin{abstract}
The article concentrates on the study of the theoretical contributions of the cuban magazine Pensamiento Crítico on the anniversary 50th of its first number. It analyzes its contributions to Latin American critical theory its relations with the process of which it arises: the Cuban Revolution. Difficult relation, if consider that de magazine was by orders of the highest authority of the country after 5 years of work. It analyzes from eight features the magazine political and theoretical praxis that give it an original and unprecedented character, that also make it be part of the revolutionary tradition and heresy of which Fidel Castro Ruz and Ernesto "Che" Guevara were part.
\end{abstract}

Keywords: Cuban Revolution, Critical Theory, Cuba, Latin American Left

La revista cubana Pensamiento Crítico apareció en febrero de 1967. Fue creada por el Departamento de Filosofía de la Universidad de La Habana -fundado en 1963, tras la reforma universitaria que volvió oficial la impartición de marxismo a todas las carreras de las universidades de La Habana, Central y Oriente - que recibió la encomienda de "incendiar el atlántico” por Osvaldo Dorticós Torrado, entonces Presidente de Cuba (DORTICÓS, 2013, p. 41). Era una época intensa, agitada, en una Revolución que conmocionó al mundo entero.

1 El presente artículo tiene como base una investigación del autor para obtener el grado de maestro en estudios latinoamericanos por la UNAM y una estancia de investigación en Cuba de octubre de 2016 a enero de 2017.3

*Licenciado en Filosofía, estudiante de la maestría en Estudios latinoamericanos por la Universidad Nacional Autónoma de México. Correo: magdielsq@gmail.com 
La Revolución cubana no fue un suceso más del siglo XX, como tampoco Pensamiento Crítico (en adelante PC) fue una revista más de tantas que existieron en esa época. Ambas responden a algo totalmente distinto. Son parte de la segunda ola de revoluciones del siglo XX con epicentro en el llamado Tercer Mundo, que abrieron un nuevo sentido en la historia oponiéndose a la subordinación y dominio imperantes.

La Revolución en la mayor isla del Caribe, muy cerca de la gran potencia imperial que se coronó tras la Segunda Guerra Mundial, se atrevió a luchar contra la dictadura y los poderes que fuera de Cuba la apoyaban. Una lucha triunfante que derribó la dictadura, conquistó el poder político, sustituyó el Estado neocolonial burgués por uno de transición socialista que sólo a tres años de su triunfo, el 16 de abril de 1961, hizo evidente que era tal, en la concentración masiva en las honras fúnebres a las víctimas de los ataques aéreos que preludiaron la fallida invasión de Playa Girón.

Una revolución que es reconocida por las potencias creadoras que desató en materia de cine, música, literatura, educación básica y sistema de salud, pero a la que pocos aportes se le reconocen en el ámbito teórico, de las ciencias sociales y la filosofía.

Una gran herejía que rompió con todas las previsiones, cálculos políticos y también con los pensamientos dominantes, incluidos los revolucionarios. Ernesto "Che" Guevara la definió como una "rebelión contra las oligarquías y los dogmas revolucionarios" ” (GUEVARA, 1978, p. 228). Como toda herejía, fue y es heredera y fundadora de una tradición disruptiva, la de los oprimidos que se levantan para barrer con toda opresión. ${ }^{2}$

El presente texto se concentra en analizar en ocho grandes aspectos de la revista PC como praxis política y teórica, que hizo de ella ser herejía y parte de una profunda tradición revolucionaria

Herejía y tradición propias no tan sólo de la revista sino de la Revolución de la que nació. Por ende, entre los rasgos expuestos se encuentran varios entrecruzamientos que caracterizan tanto a la revista como al proceso social que la produjo. La revista en todo caso, es en este texto una ventana para mirar a través de ella, lo que una determinada posición dentro de la Revolución pensó y produjo. Ventana que suele ser ignorada, no tanto por el desenlace prematuro que tuvo $P C$ - del que hablaremos al final del texto -, sino porque el pensamiento que produjo la Revolución cubana suele ser poco analizada y menos aún esa vertiente crítica que, bajo el liderazgo de Fidel Castro Ruz y Ernesto "Che" Guevara, la hacen difícil de asirse a simples homenajes, porque se niega ser un elemento del pasado, bajo la premisa de la actualidad de la revolución. ${ }^{3}$

2 "La historia no sólo tiene la tarea de hacerse de la tradición de los oprimidos, sino también de fundarla" (BENJAMIN, 2005, p. 57).

3 La actualidad de la revolución fue definida por György Lukács, en el plano teórico, como "el estudio de todos y cada uno de los problemas particulares del momento en su concreta relación con la totalidad histórico-social; su consideración como momentos de liberación del proletariado" (LUKÁCS, 1979, p. 14). Esto es, que en el plano de la acción política, asumir que "la revolución se ha convertido en el problema crucial" (LUKÁCS, 1979, p. 13) y por 
El texto no agota las reflexiones necesarias sobre la revista, intenta ser parte de ellas. Las visiones expuestas en el coloquio: "Con arreglo a esta opinión trabajaremos" A 50 años de Pensamiento Crítico realizado en la Habana, Cuba, el 21 de febrero de 2017 han sido un primer espacio de reflexión importantísimo y abundan en otros elementos que el presente no lo hace.

Doce mil páginas, más de seiscientos textos distribuidos en 53 números, a lo largo de cinco años y cientos de miles de ejemplares distribuidos en Cuba y el mundo quedan como el testimonio palpable de lo que fue la revista Pensamiento Crítico. A continuación, expongo los ocho rasgos característicos de la revista como herejía y tradición.

1. Desarrolló una posición original en los debates de la época y logró influir fuera de Cuba. La fuerza del proceso social del cual era parte $P C$ permitió que el acercamiento de muchos pensadores pudiese tener un rasgo específico, y en cierta manera inédito: sus aportes tenían que servir a la revolución en marcha.

Fue capaz de relacionarse con diversos intelectuales del mundo, aprendió y debatió con ellos, apropiándose críticamente de las ideas y yendo más lejos en sus formulaciones, sin ser pensamientos colonizados. ${ }^{4}$ Colaboraron en la revista Regis Debray, Louis Althusser, Jean Paul Sartre, Giovanni Arrighi, Michael Löwy, Nicos Poulantzas, Étienne Balibar, Rudi Dutschke, Eric Hobsbawm, Ralph Miliband, André Gunder-Frank y otros intelectuales europeos. Los norteamericanos Leo Huberman, Paul Baran, Paul Sweezy, Harry Magdoff, entre otros. Intelectuales latinoamericanos como Aníbal Quijano, Gerard Pierre Charles, Theotonio Dos Santos, Antonio García, Segio Bagú, y otros. Destacados revolucionarios de Asia, África y América Latina como Fabricio Ojeda, Amílcar Cabral, Malcom X, Roque Dalton, Turcios Lima, Carlos Marighela. Además, dio a conocer en español textos teóricos entonces desconocidos -y que tras llegar a ser considerados como sumamente relevantes, raramente se les atribuye su primera publicación a $P C$ - como los de Gunder-Frank, Sergio Bagú, Karl Korsch, György Lukács, Antonio Gramsci, Vladimir I. Lenin y Ho Chi Minh.

Los productos intelectuales que se recuperaban no le daban sentido a la revista, la revista tenía sentido propio. Las contribuciones de otras revistas tenían por objeto apropiarse de los debates del momento, intervenir críticamente y fijar una posición propia. ${ }^{5}$ Además de los

ende, no hay "campo alguno de la lucha de clases en el que no vengan contenidas posibilidades revolucionarias (o contrarrevolucionarias)" (LUKÁCS, 1979, p. 121) en todo momento y no sólo en las coyunturas favorables o la crisis terminal.

4 Usamos aquí la expresión pensamiento colonizado en el sentido que lo hace Fernando Martínez Heredia, quien fuera integrante del Departamento de Filosofía y su director desde mediados de 1963 hasta 1967; fue también director de la revista $P C$ : "El pensamiento colonizado porta enfermedades disímiles, pero complementarias: creencia en una superioridad inherente a los países "desarrollados", manifiesta en muchos campos, modelos a imitar y cima inalcanzable, "vanidad, deseo de ser aceptado por "ellos" y autosubestimación"; consumo de principios abstractos, asimilación y reproducción de los principios abstractos, ideas y productos culturales de las metrópolis, "no exento de angustia porque casi nunca logra estar al día" (MARTÍNEZ, 2015, p. 228).

5 "Vamos a hacer una revista "seria", más grandota, que influya de otro modo". A fines de 1966 organizamos y comenzamos aquel trabajo. El primer título que pensamos era muy desabrido - Revista de Revistas-, por suerte 
teóricos y revolucionarios con los que se articuló, tuvo un incesante intercambio -organizados por Jacinto Valdés-Dapena Vivanco- con 92 publicaciones, entre las que destacan: New Left Review, Social Register, Monthly Review, Partisans, Les Temps Modernes, Punto Final, Pasado y Presente.

2. Trabajó de modo embrionario una propuesta original de interpretación de la revolución cubana como parte de los trabajos del Departamento de Filosofía. ${ }^{6}$ Por ser un aporte a la acción y al pensamiento político de ese proceso necesariamente se enfrentó a las interpretaciones dominantes - aún en sus vetas críticas - y esbozó el estudio de las características de la Revolución y las raíces de las cuales provenía. Destacan "El Moncada, asalto al futuro" de Germán Sánchez Otero en el que se analiza el asalto al cuartel Moncada "por su contenido y nutrientes histórico" la fecha clave del proceso revolucionario y para su ulterior desarrollo (SÁNCHEZ OTERO, 1969). El texto "La Revolución pospuesta" de Ramón de Armas-quien luego se convertiría en uno de los más grandes historiadores contemporáneos de Cuba-en el que analiza desde la guerra de independencia la imposibilidad de la burguesía para ser clase nacional, el nacimiento de una neocolonia desde la colonia y la ocupación norteamericana y la contraposición del proyecto martiano (DE ARMAS, 1971). Pedro Pablo Rodríguez - hoy director del proyecto de edición crítica de las obras completas de José Martí - publicó, en el mismo número que De Armas, un análisis del proyecto martiano y la independencia como una revolución de liberación nacional que trascendió el horizonte bajo el que se suelen juzgarse los objetivos y alcances políticos de estas gestas de la región y estudió las características de la organización y guerra revolucionaria en la perspectiva de la fundación de una nueva república (RODRÍGUEZ, 1971). También la publicación de diversos materiales de la historia cubana de y sobre revolucionarios cubanos. El número 39 de la revista se convirtió en uno de los documentos obligados para conocer la Revolución cubana de los años 30, en la que se demuestra a través de documentos el nacimiento del socialismo cubano y de los desafíos históricos socialistas, de liberación nacional y anti imperialistas que sólo la revolución dirigida 30 años después por Fidel Castro logrará enfrentar. ${ }^{7}$

La interpretación histórica esbozada en $P C$ plantea los vínculos y relaciones con las revoluciones cubanas previas como las bases ideológicas fundamentales de las que se nutrió la del 59, en esto confrontó a la consideración de que era una revolución sin ideología como expuso Jean Paul Sartre en Huracán sobre el Azúcar (SARTRE, 1960). Ligado a esto, y con

no se llamó así. Respondía a la idea de hacer una revista para publicar lo más interesante que encontráramos por ahí, propósito que fue superado enseguida. discutíamos mucho. Pensamiento Crítico fue un nombre feliz, porque atañía a las cuestiones fundamentales: al pensamiento y a la crítica. No es que fuéramos brillantes. Nos alivió tener al fin un título." (MARTÍNEZ, 2010b, p. 12).

6 A pesar de ser el gran suceso de la historia cubana no existen muchos escritos en torno a esto. Los materiales más abundantes son testimonios personales. Apenas ahora, en especial con los investigadores de Oriente, Mario Mencía, Reynaldo Suárez y Frank Josué Solar están apareciendo nuevos materiales de interpretación histórica.

7 En especial Pensamiento Crítico 1970 (Cuba: La Habana) № 39, abril. Dedicado por completo a esta Revolución y con 407 páginas. 
base en los planteamientos políticos, la posición de sus dirigentes y el tipo de medidas que se implementaron - más allá de las referencias explícitas que se hicieran sobre ella- plantea que se trata, desde sus primeros momentos, de una revolución en la que la liberación nacional y el socialismo son una unidad que niega las etapas y rompe con la visión marxista tradicional que sostiene como necesario -para países "atrasados" - el tránsito por una revolución democrática burguesa para luego arribar al socialismo, como Vania Bambirra expone en La Revolución Cubana: Una reinterpretación (BAMBIRRA, 1976). En el número 36, de enero de 1970 se reproduce un discurso de Fidel Castro que bajo el título "Hoy, para el mundo subdesarrollado, el socialismo es condición de desarrollo" (CASTRO, 1968b) fundamenta la visión cubana de socialismo, en la que el factor subjetivo está por encima de las determinaciones económicas de desarrollo. En otro discurso de Fidel se hace una reconstrucción histórica en la que define el curso de la historia no son las etapas ni los cambios de modo de producción, sino la unidad de las luchas del pueblo cubano por buscar su independencia y justicia social, que sólo se pueden alcanzar con la revolución socialista iniciada en 1959.

En este plano, la herejía no se reduce a esbozar las líneas de una interpretación original propia, sino que ella tenía -y aún sigue teniendo - consecuencias en el plano de la acción política, para quienes se planteaban desatar procesos de ese tipo en sus países.

3. Subvirtió las relaciones entre trabajo teórico y militancia política ${ }^{8}$ en al menos tres aspectos:

a) Rompieron con las formas convencionales de relación entre trabajo manual e intelectual, trabajo teórico y preparación militar. Todos los que participaban del armado, contenidos y redacción tenían una actividad política más allá de la revista, desarrollaban tareas de defensa de la revolución, impartían cursos, participaban en los cortes de caña.

b) Desarrollaron un marxismo crítico (ver punto 6), sin abandonar el movimiento revolucionario. ${ }^{9}$

c) Asumieron la defensa del proceso revolucionario sin sujetarse a una obediencia ciega. Y cuando resultó imposible sostener la publicación (vid.infra), sortearon las adversidades.

\footnotetext{
8 Este reto lo expusieron en su primer número con estas palabras: "Opinamos que el intelectual revolucionario es, ante todo, un revolucionario a secas, por su posición ante la vida; después, aquel que crea o divulga según su pasión y su comprensión de la especificidad y el poder transformador de la función intelectual. Si la primera condición existe, le será fácil coincidir con la necesidad social. Con arreglo a esta opinión trabajaremos" "Presentación" 1967 Pensamiento Crítico 1967 (Cuba: La Habana) No 1, febrero: 2.

9 En la "Presentación" del número 41, hacen referencia al caso de Karl Korsch y György Lukács. El primero, luego de una brillante trayectoria intelectual abandonó el movimiento comunista; el segundo, claudicó "en sucesivas autocríticas que no ayudaron nada al desarrollo del sentido de los deberes del intelectual comunista en la dictadura del proletariado" Cfr. Pensamiento Crítico 1970 (Cuba: La Habana) oo 41, junio: 7
} 
4. Alcanzó un amplísimo número de lectores y logró llegar a la gente común para que fuese una herramienta de lucha a través de una propuesta integral novedosa. El primer número constó de 4 mil ejemplares, el 2/3 de seis mil, el 5 de diez mil y los demás números de 15 mil. La revista llegaba a agotarse en los primeros días (MARTÍNEZ, 2010b, p. 12).

Nunca se constriñó a ser una difusora de ideas procesadas ni consideró a sus lectores como inferiores. No subestimó a las personas ni a las masas que hacían la revolución, sino que trató de ponerse a su altura, debatir con ellas y darles un instrumento de lucha. Ejemplo de esto, un colaborador de la revista la intercambiaba con su boleador de zapatos, que siempre estaba pendiente de ella. ${ }^{10}$

Se planteó hablar el lenguaje necesario para la revolución que se hacía, posición contraria a las vulgarizaciones del marxismo tradicional. Cuestión consecuente al mismo tiempo con el debate sobre el uso de manuales en la enseñanza de filosofía que sostuvo el Departamento de Filosofía con intelectuales de la revista Teoría y Práctica (cuyos intelectuales provenían del Partido Socialista Popular). ${ }^{11}$

La revista tenía también una propuesta estética original con el diseño de Alfredo Rostgaard. Como expresa Fernando Martínez Heredia - director de la revista y miembro del Departamento de Filosofía-: "la dimensión artística no era ajena a la revista, sino uno de sus aspectos principales” (MARTÍNEZ, 2010b, p. 13).

Era perseguida y quemada en dictaduras y gobiernos "democráticos" porque era un instrumento de los revolucionarios. Se leía en la cárcel de Lecumberri, en México; por militantes revolucionarios de Chile, Brasil, Perú, Uruguay; por la dirección del Frente Sandinista de Liberación Nacional de Nicaragua (MARTÍNEZ, 2010b, p. 18).

5. Descubrió a través de sus primeras obras a jóvenes que luego serían considerados grandes intelectuales. No se trata, ciertamente, de un descubrimiento, sino que la revista les demandó y posibilitó ir más allá de sí mismos.

Por nombrar algunos ejemplos: "Del antiguo apogeo a la humillación de nuestro tiempo" es un relato de la guerra de Paraguay escrita por el entonces desconocido Eduardo Galeano. ${ }^{12}$ Mientras John Saxe-Fernández publicaba uno de sus primeros escritos sobre el imperialismo. ${ }^{13}$

10 Entrevista del autor con Pedro Pablo Rodríguez López. Cuba: Centro de Estudios Martianos, El Vedado, La Habana, jueves 1 de diciembre de 2016.

$11 \mathrm{El}$ debate es importantísimo al momento de revisar la historia del pensamiento crítico cubano. La polémica sobre los manuales se dio en la revista Teoría y Práctica, en sus números 28, 30, 31 y 31, de los años 1966-1967. Los polemistas fueron, Humberto Pérez y Felix de la Uz, por la revista Teoría y Práctica y por el departamento de Filosofía Aurelio Alonso. Posteriormente fue compilada por el Departamento de Filosofía en 1968 Lecturas de filosofía (Tomo II) (Cuba: La Habana, Cuba, Instituto del Libro): 745-776.

12 Pensamiento Crítico 1971 (Cuba: La Habana) No 51, abril: 215-227

13 "Hacia un modelo de la estrategia militar norteamericana" 1970 Pensamiento Crítico 1970 (Cuba: La Habana) No 44 , septiembre: 7-55 
Jesús Díaz realizó un importante estudio sobre Lenin. ${ }^{14}$ Fernando Martínez Heredia publicó "Marx y el origen del marxismo", ${ }^{15}$ un texto de una riqueza y rigor inconmensurables; uno de sus primeros textos públicos en los que muestra no sólo una lectura original del pensamiento de Karl Marx, sino las bases de desarrollo de un marxismo original, que se piensa desde el contexto histórico y bajo la luz de la revolución. También están los textos de estudiantes y luego profesores del Departamento de Filosofía -que mencionamos en el punto 2 - como Ramón de Armas y Pedro Pablo Rodríguez cuyos trabajos de interpretación histórica y de estudio del pensamiento martiano, respectivamente serían fundamentales. Germán Sánchez Otero, reconocido político e intelectual tendría también ahí publicado uno de sus primeros textos.

6. Gestó las bases de un marxismo de nuevo tipo y de una práctica política revolucionaria ajena a la posición del bloque socialista. La formulación "tenemos que hacer que el 'marxismoleninismo' se ponga a la altura de la Revolución cubana" (MARTÍNEZ, 2010a, p. 53), hecha por Fernando Martínez Heredia y asumida como propia por el Departamento de Filosofía guió el trabajo de la revista.

La ruptura teórica que planteó Pensamiento Crítico - por nombrar sólo a la revista, aunque esto no se comprende sin el Departamento de Filosofía que la creó- formuló las bases de un marxismo "de nuevo tipo". Un marxismo original, distante del dogma oficial, la ortodoxia contaba con un enorme poder y prestigio. Al mismo tiempo, tomó distancia de otras corrientes que, aunque críticas del marxismo soviético, no rompían del todo con sus presupuestos. La confianza en que la contradicción entre fuerzas productivas y relaciones de producción echen por la borda a la sociedad existente, la necesidad del desarrollo histórico según la ley de valor (expresión refinada del etapismo), "el ser social determina la conciencia social" elevado a dogma, o la aseveración de la imposibilidad de realizar el socialismo por falta de correspondencia adecuada entre las relaciones de producción y las fuerzas productivas, y otros presupuestos correspondientes al marxismo soviético, y extendido -en algunos casos- a marxismos críticos, serán enfrentadas por la revista, pues en ellas subyacen concepciones propias del pensamiento burgués como progreso, linealidad, civilización, y por ende, no logran abrir un horizonte de superación de la sociedad. El número 41, de junio de 1970, se concentra en refutar esta idea, los textos "Marx y el origen del marxismo" y "Los conceptos del marxismo determinista"-de FMH y Jorge Gómez Barranco, respectivamentefueron preparados a partir de los grupos de estudio del Departamento y son ellos -junto los textos de György Lukács y Karl Korsch - en donde mejor se puede ubicar la distancia crítica respecto al marxismo dogmático y apreciar los rasgos de ese marxismo de nuevo tipo, que toma como bases el suelo del socialismo cubano en contraposición con el socialismo de origen soviético.

14 "El marxismo de Lenin" en Pensamiento Crítico 1970 (Cuba: La Habana) № 38, marzo: 6-60 15 En Pensamiento Crítico 1970 (Cuba: La Habana) No 41, junio: 10-47. El autor sostiene un proyecto inacabado sobre el pensamiento de Karl Marx. 
7. Se apropió y se hizo parte de la tradición socialista cubana de liberación nacional y de su pensamiento, que se inscribe históricamente como síntesis y superación de las diversas vertientes del pensamiento social cubano que precedieron a la Revolución del 59. ${ }^{16}$

La revista colocó en la dimensión teórica las producciones de Fidel Castro y Ernesto "Che" Guevara, a quienes se les subestimó desde el marxismo soviético y en Cuba por los militantes del Partido Socialista Popular, por considerarlos aventureros o reducir su aporte a liderazgos tan sólo políticos. Se publicaron 35 textos del Che y 17 de Fidel, en los que se recupera su visión de la historia de Cuba y exponen sus ideas fundamentales sobre la revolución socialista y la liberación nacional (PONCE, 2017). Tras la muerte del "Che" se publicaron dos números ${ }^{17}$ a través de los cuales se le mostraba como un teórico de la Revolución cubana y mundial; un dirigente político subversivo e internacionalista; un comunista y un guerrero. Hazaña que sólo volvería a replicarse a fines de los ochenta -tras el esfuerzo de Fidel Castro por abrir un periodo de rectificación de errores y tendencias negativas- con textos de Carlos Tablada, Fernando Martínez Heredia y otros. También se publicó el inédito "Mi campaña con el Che" de Inti Peredo que abre con una polémica con el Partido Comunista de Bolivia. ${ }^{18}$

Fidel Castro fue mostrado por la revista a través de algunos textos del Movimiento 26 de Julio, ${ }^{19}$ discursos como el del Congreso Cultural de La Habana ${ }^{20}$ y del Primer Congreso Nacional de Educación y Cultura ${ }^{21}$ y otras posiciones sobre la cultura y la historia de Cuba como bases para el desarrollo del socialismo; ${ }^{22}$ reflexiones distantes al socialismo soviético en relación con los países del tercer mundo ${ }^{23}$ y diversos discursos, ${ }^{24}$ que en su conjunto muestran a Fidel como un educador popular y el más importante teórico y revolucionario cubano. ${ }^{25}$ De esto se puede

16 El liberalismo, el patriotismo, el anti imperialismo, el democratismo, las ideas de justicia social fueron asumidas desde el socialismo, tras el triunfo de la revolución (MARTÍNEZ, 2010a).

17 Cfr. Pensamiento Crítico 1967 (Cuba: La Habana) No 9, noviembre y Pensamiento Crítico 1968 (Cuba: La Habana) No 14, marzo, respectivamente.

18 Pensamiento Crítico 1971 (Cuba: La Habana) No 52, mayo: 2-67

19 Cfr. Pensamiento Crítico (Cuba: La Habana), números 6, julio de 1967; 21, octubre de 1968; 31, agosto de 1969 20 Cfr. Pensamiento Crítico 1968 (Cuba: La Habana) No 12, enero: 5-27.

21 Este número coincidió con el cierre de la revista. Para no ver frustrada su publicación recurrieron a la revista amiga Referencias 1971 (Cuba: La Habana) No 3, volumen 2. Para el autor: La revista Referencias fue fundada por la dirección del Partido Comunista de Cuba de la Universidad de La Habana y su director fue un miembro de PC: José Bell Lara.

22 "Si las raíces y la historia de este país no se conocen, la cultura política de nuestras masas no estará suficientemente desarrollada" (Castro, 1968: 181-216).

23 Cfr. Pensamiento Crítico 1970 (Cuba: La Habana) No 36, enero: 133-185

24 Cfr. Pensamiento Crítico 1970 (Cuba: La Habana) № 45, octubre

Pedro Pablo Rodríguez 1971 mayo.1969 s.) agazine was ista fue cerrada, luego de 5 años de trabajo, por .

25 En una tradición que se caracteriza por la humildad, el reconocimiento explícito a Fidel se guardó por hasta su muerte. Dos textos publicados tras la muerte de Fidel que reflejan a mi parecer de mejor manera el legado de Fidel para el presente, son de Fernando Martínez Heredia: 2017 "Claves del anticapitalismo y el antimperialismo hoy. Las visiones de Fidel en los nuevos escenarios de lucha” (MARTÍNEZ, 2017) y "Fidel Vive” (MARTÍNEZ, 2016). 
comprender la formulación "Fidel puso el marxismo en español" (MARTÍNEZ, 2011) que haría años después el director de $P C$.

Recuperó también a revolucionarios como Julio Antonio Mella, Antonio Guiteras, los hermanos Saíz Montes de Oca ${ }^{26}$ y Frank País.

8. Planteó un pensamiento y una política desde el Tercer Mundo, consecuente con el internacionalismo cubano que se gestó en la época. En ese sentido:

a) Recuperó y publicó textos de luchas de América Latina, Asia y África (los primeros tres números iban dedicados a cada una de estas regiones del mundo); documentos de la revolución palestina, ${ }^{27}$ las luchas revolucionarias del movimiento negro en Estados Unidos también fueron rescatados. $^{28}$

b) Difundió textos de Franz Fanon, Amílcar Cabral y otros pensadores claves para comprender el mundo desde los países que se proponen acabar con el colonialismo y el neocolonialismo.

c) Organizó reflexiones de primer orden sobrela estrategia revolucionaria internacionalista y el problema de la violencia. En esto destacan las reflexiones de los revolucionarios brasileños en torno a la lucha, ${ }^{29}$ los textos del "Che", Camilo Torres, Fabricio Ojeda y Z. Martin Kowalewsky. ${ }^{30}$

Pensamiento Crítico fue una expresión de tradición y herejía de la Revolución cubana, un acumulado de la riqueza teórica y política del proceso histórico más profundo, radical, opuesto y diferente al orden capitalista en América Latina. Sin embargo, las relaciones entre la Revolución y el Departamento -y por supuesto la revista- demuestran las contradicciones y dificultades de un proceso para ir más allá de lo alcanzado. En la medida que Cuba no logró desatar una autonomía económica y que el asedio de Estados Unidos se hacía más fuerte, la influencia de la URSS sobre Cuba fue mayor en todos los ámbitos de la vida. Los señalamientos que a lo largo de los años habían hecho los ex militantes del PSP y los autores del proceso de la Microfracción ${ }^{31}$ contra el Departamento y PC habían sido sorteados sin grandes dificultades por el grupo, el rector de la Universidad de La Habana -José Miyar Barruecos- y el propio Fidel Castro -a quien señalaban como el protector del grupo- fueron más difíciles de soportar en la medida que la URSS ganaba peso al interior de Cuba. La producción de la revista se interrumpió en un momento en que todavía tenía un camino por andar

26 Cfr. Pensamiento Crítico 1968 (Cuba: La Habana) No 22, noviembre.

27 Pensamiento Crítico 1970 (Cuba: La Habana) № 40, mayo.

28 Pensamiento Crítico 1968 (Cuba: La Habana) No 17, junio.

29 Pensamiento Crítico 1970 (Cuba: La Habana) No 37, febrero y Pensamiento Crítico 1970 (Cuba: La Habana) No 46 , noviembre.

30 De este último el texto "El papel de la guerra revolucionaria en el desarrollo de la cultura" (KOWALEWSKY, 1969 , p. 59-82).

31 Una conspiración dentro de la revolución por ex militantes del PSP liderados por Aníbal Escalante para adueñarse de la conducción del proceso y presuntamente apoyados por la URSS. 
el último número salió en junio de 1971 y el Departamento fue cerrado a fines de ese mismo año. Un año después el rector de la Universidad fue removido. Fernando Martínez Heredia, quien falleció el 12 de junio de 2017, explicó el cierre como "una tragedia en la que las necesidades del Estado parecían más decisivas que los criterios ideológicos o teóricos" (MARTÍNEZ, 2010a, p. 40).

La necesidad de conservar la unidad y la de resolver las dificultades económicas resultaron más fuertes que el desafío crítico abierto por el Departamento. El golpe a esta iniciativa que se hizo parte de la tradición y herejía cubana revolucionaria impactó profundamente en el ámbito. El golpe a esta iniciativa que se hizo parte de la tradición y herejía cubana revolucionaria impactó profundamente en el ámbito intelectual y varias generaciones se vieron privadas no sólo de ser partícipes de esta experiencia, sino de conocerla, pues privó el silencio en torno a ella por muchos años. Sólo de modo reciente, empieza a contarse la historia y a recuperarse la herencia. Materiales de hace 50 años empiezan a ser aprehendidos y su encuentro sorprende a las nuevas generaciones por su pertinencia y actualidad. Los jóvenes empiezan a hacer suya esa historia y sueñan con hacer su propia herejía.

Ahondar en las repercusiones, el contexto y las contradicciones en el proceso revolucionario que llevaron al fin de la revista son cuestiones que rebasan los objetivos del presente. Empero, es una tarea pendiente y necesaria de ser desarrollada.

Así como la revista y la Revolución de la que nació no pueden explicarse por correspondencia con un estadio del desarrollo capitalista sino por la violentación subjetivaconsciente de las leyes del capitalismo, recuperarle es sumergirse en una herejía y tradición que está en riesgo de desaparecer si el presente no se reconoce como aludido en ellas (BENJAMIN, 2005. p 20). Incluso la Revolución Cubana está en tensión permanente - y más aguda en los últimos años - entre afirmarse y profundizarse como revolución o transitar a ser una posrevolución. ${ }^{32}$ La solución de esa tensión trasciende el ámbito teórico. Vendrá de la acción de las y los herederos de la misma.

Desde fuera de Cuba, mirar a través de la ventana que nos ofrece Pensamiento Crítico es ir más allá del conocer su historia, sus textos, y sobre todo asumir - desde nuestro suelo y circunstancias - esa invitación pendiente que la herejía y la tradición nos hicieran un primero de enero de 1959.

\section{Bibliografía}

BAMBIRRA, Vania. La revolución cubana, una reinterpretación. México: Editorial Nuestro Tiempo, 1976. BELL, José; LÓPEZ, Delia Luisa; CARAM, Tania (comps). Documentos de la revolución Cubana 1965. Cuba, La Habana: Ciencias Sociales, 2013).

BENJAMIN, Walter. Tesis sobre la historia y otros fragmentos. México: Editorial Contrahistorias, 2005.

CASTRO, Fidel. La Historia me absolverá (fragmento) Pensamiento Crítico. Cuba: La Habana, n.6, julio 1967, p. 3-7

32 Este asunto es de prima importancia, superior a este texto. Aquí apenas lo enuncio. Sobre esto se puede leer el texto de Fernando Martínez Heredia “Días históricos. Épocas históricas” (2015). 
Los aniversarios del 26 de julio (fragmento) Pensamiento Crítico. Cuba: La Habana, n. 6, julio 1967, p. 49-71

Discurso clausura Congreso Cultural de La Habana. Pensamiento Crítico. Cuba: La Habana, n. 12, enero 1968a, p. 5-27

Si las raíces y la historia de este país no se conocen, la cultura política de nuestras masas no estará suficientemente desarrollada. Pensamiento Crítico. Cuba: La Habana, n. 20, septiembre 1968b, p. 181-216

Manifiesto $N^{o} 1$ del 26 de Julio al pueblo de Cuba. Pensamiento Crítico. Cuba: La Habana, n.21, octubre 1968, p. 207-220

Manifiesto $N^{\circ} 2$ del 26 de Julio al pueblo de Cuba. Pensamiento Crítico. Cuba: La Habana, n.21, octubre 1968d, p. 221-227

El movimiento 26 de julio. Pensamiento Crítico. Cuba: La Habana, n.31, agosto 1969, p. 3-12

1970 Hoy, para el mundo subdesarrollado, el socialismo es condición de desarrollo. Pensamiento Crítico. Cuba: La Habana, n.36, enero 1970, p. 133-185

Discurso de Clausura del Primer Congreso Nacional de Educación y Cultura. Referencias. Cuba: La Habana, v. 2, n.3, 1971, p. 157-166.

DE ARMAS, Ramón. La Revolución pospuesta. Pensamiento Crítico. Cuba: La Habana, n. 49-50, febrero 1971, p. 7-119. DÍAZ, Jesús. El marxismo de Lenin. Pensamiento Crítico. Cuba: La Habana. n. 38, marzo 1970, p. 6-60

DORTICÓS, Osvaldo. Lo más importante: que ustedes enseñen a pensar a los alumnos. In: BELL, José; LÓPEZ, Delia Luisa y CARAM, Tania (comps.) Documentos de la Revolución Cubana 1965. Cuba, La Habana: Ciencias Sociales, 2013.

GALEANO, Eduardo. Del antiguo apogeo a la humillación de nuestro tiempo. Pensamiento Crítico. Cuba: La Habana, n.51, abril 1971, p. 215-227

GUEVARA, Ernesto. El diario del Che en Bolivia. México: Editorial Siglo XXI, 1978.

KOWALEWSKY, Z. Martin. El papel de la guerra revolucionaria en el desarrollo de la cultura. Pensamiento Crítico. Cuba: La Habana, n. 28, mayo 1969, p. 59-82

Lecturas de filosofía (Tomo II). Cuba: La Habana, Cuba, Instituto del Libro, 1968.

LUKÁCS, György. Lenin (La coherencia de su pensamiento). México: Editorial Grijalbo, 1979.

MARTÍNEZ HEREDIA, Fernando. Marx y el origen del marxismo. Pensamiento Crítico. Cuba: La Habana, n. 41, junio 1970, p. 10-47

Socialismo. México: Ocean Press-Ocean Sur, 2008. (Col. Pensamiento socialista).

El ejercicio de pensar. Cuba: Ruth Casa editorial y Ciencias Sociales, 2010a.

La crítica en tiempos de Revolución. Antología de textos de Pensamiento Crítico. Cuba: Santiago de Cuba, Editorial Oriente, 2010b.

Fidel puso al marxismo en español. Síntesis de las ideas expresadas en la Mesa Redonda de Telesur: Vida y luchas de Fidel Castro transmitida el jueves 11 de agosto. Cuba: La Habana, 2011. Disponível em: <http://www.cubadebate.cu/opinion/2011/08/13/fidel-puso-al-marxismo-en-espanol/\#.WMBNLxCGl4g> Acceso em: 20 dec. 2016. . A la mitad del camino. Cuba: La Habana, Editorial de Ciencias Sociales, 2015.

Fidel Vive. Texto con motivo de la muerte de Fidel Castro Ruz el 25 de noviembre de 2016. Cuba: La Habana, 28 de noviembre de 2016. Disponível em: <http://www.cubadebate.cu/noticias/2016/11/28/fernando-martinez-heredia-fidel-vive/> Acceso em: 28 nov. 2016.

Claves del anticapitalismo y el antimperialismo hoy. Las visiones de Fidel en los nuevos escenarios de lucha. Cuba: La Habana,11 de enero de 2017. Disponível em: < http://www.cubadebate.cu/especiales/2017/03/07/ las-visiones-de-fidel-en-los-nuevos-escenarios-de-lucha/>. Acceso em: 7 mzo. 2017

Pensamiento Crítico. Cuba: La Habana, n. 1, febrero 1967. 
Pensamiento Crítico. Cuba: La Habana, n. 6, julio 1967.

Pensamiento Crítico. Cuba: La Habana, n. 9, noviembre 1967.

Pensamiento Crítico. Cuba: La Habana, n. 12, enero 1968.

Pensamiento Crítico. Cuba: La Habana, n. 14, marzo 1968.

Pensamiento Crítico. Cuba: La Habana, n. 17, junio 1968.

Pensamiento Crítico. Cuba: La Habana, n. 20, septiembre 1968.

Pensamiento Crítico. Cuba: La Habana, n. 21, octubre 1968.

Pensamiento Crítico. Cuba: La Habana, n. 22, noviembre 1968.

Pensamiento Crítico. Cuba: La Habana, n. 28, mayo 1969.

Pensamiento Crítico. Cuba: La Habana, n. 31, agosto 1969.

Pensamiento Crítico. Cuba: La Habana, n. 36, enero 1970.

Pensamiento Crítico. Cuba: La Habana, n. 37, febrero 1970.

Pensamiento Crítico. Cuba: La Habana, n. 38, marzo 1970.

Pensamiento Crítico. Cuba: La Habana, n. 39, abril 1970.

Pensamiento Crítico. Cuba: La Habana. n. 40, mayo 1970.

Pensamiento Crítico. Cuba: La Habana, n. 41, junio 1970.

Pensamiento Crítico. Cuba: La Habana, n. 44, septiembre 1970.

Pensamiento Crítico. Cuba: La Habana, n. 45, octubre 1970.

Pensamiento Crítico. Cuba: La Habana, n. 46, noviembre 1971.

Pensamiento Crítico. Cuba: La Habana, n. 49-50, febrero 1971.

Pensamiento Crítico. Cuba: La Habana, n. 51, abril 1971.

Pensamiento Crítico. Cuba: La Habana, n. 52, mayo 1971.

Pensamiento Crítico. Cuba: La Habana, n. 53, junio 1971.

PEREDO, Inti. Mi campaña con el Che. Pensamiento Crítico. Cuba: La Habana, n. 52, mayo 1971, p. 2-67

PONCE, Vilma N. Suárez. Una mirada métrica a la revista Pensamiento Crítico. In: Anales de Investigación, $\mathrm{n}$.

3. Cuba: La Habana. Enero-diciembre 2007, p. 102-138

Referencias. Cuba: La Habana, v. 2, n. 3, 1971.

RODRÍGUEZ, Pedro Pablo. La idea de liberación nacional en José Martí. Pensamiento Crítico. Cuba: La Habana, n. 49-50, febrero 1971 , p. $120-170$

SAÍZ MONTES DE OCA, Luis. La generación del Centenario. Pensamiento Crítico. Cuba: La Habana, n. 22, noviembre 1968, p. 239-241

¿Por qué Luchamos? Pensamiento Crítico. Cuba: La Habana, n. 22, noviembre 1968, p. 242-259

SAÍZ MONTES DE OCA, Sergio. Elegía a Karl Marx. Pensamiento Crítico. Cuba: La Habana, n. 22, noviembre 1968 , p. 260

SANCHEZ OTERO, Germán, El Moncada: asalto al futuro. Pensamiento Crítico. Cuba: La Habana. n. 31, agosto 1969, p. 99-131

SARTRE, Jean Paul. Huracán sobre el azúcar. Uruguay: Montevideo, Ediciones Uruguay, 1960, 147p.

SAXE-FERNANDEZ, John. Hacia un modelo de la estrategia militar norteamericana. Pensamiento Crítico. Cuba: La Habana. n. 44, septiembre 1970, p. 7-55 